\title{
MRI may predict the onset of Alzheimer disease
}

Cortical thinning in specific brain regions may predict the onset of Alzheimer disease (AD) up to a decade before the appearance of clinical symptoms, according to new data published in Neurology. Brad Dickerson and colleagues have identified an MRI signature that can predict an individual's relative risk of developing $\mathrm{AD}$ dementia.

\section{4 ...[55\% of people] who had below-average cortical thickness ...developed AD dementia 77}

The MRI signature is derived from measurements of cortical thickness in nine brain regions known to show atrophy in patients with mild AD. Dickerson and colleagues believe that cortical thinning at these sites may be predictive of the onset of $\mathrm{AD}$ dementia. "Many older adults who are cognitively normal harbor the pathology of AD in their brains," explains Dickerson. "The next logical question was, then, would this measure be useful to predict the risk of and time to onset of dementia in cognitively normal older adults?"

The investigators assessed data from 65 elderly individuals who had participated in two separate longitudinal MRI studies. All were initially cognitively normal, according to clinical evaluations, neuropsychological tests and memory $Z$-scores. At the end of follow-up (mean 8 years), 25 individuals in each group were still cognitively normal, whereas the rest (seven patients in one study and eight in the other) had developed probable AD dementia. The investigators observed subtle but consistent cortical thinning in the individuals with probable $\mathrm{AD}$ dementia compared with those who remained cognitively normal.

Six of 11 people (55\%) who had belowaverage cortical thickness at the start of the study developed $\mathrm{AD}$ dementia during the subsequent decade. By contrast, only nine of 45 patients (20\%) with average cortical thickness and none of the nine patients with above-average cortical thickness developed the disease. Patients in the below-average cortical thickness group were also predicted to progress to dementia significantly faster than those in the average cortical thickness group. "We don't yet know whether measures of brain structure or function in living individuals with markers of $\mathrm{AD}$ pathology can be used to predict the risk of and time to the development of symptoms," says Dickerson, "but this MRI marker appears to be able to do so in a reliable fashion."

Dickerson hopes that this MRI signature will assist investigators in identifying the asymptomatic individuals who are at the highest risk of cognitive decline and dementia within the next 5-10 years. "This would be the ideal population to enroll

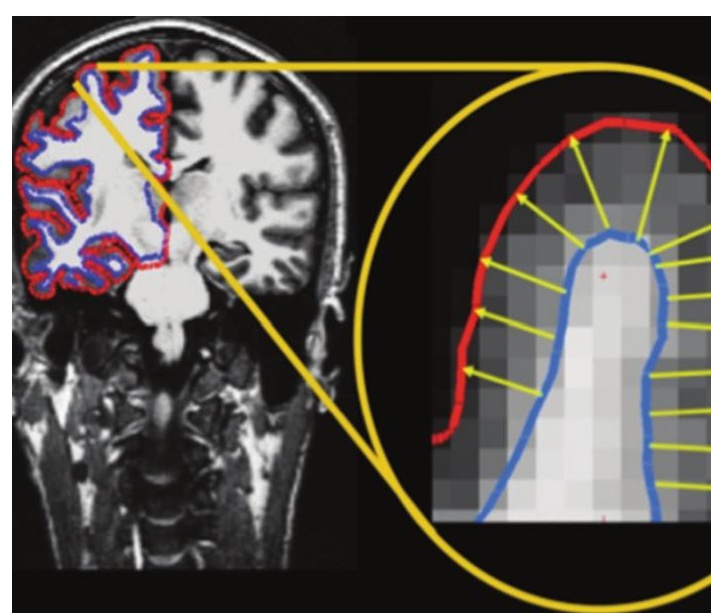

Computational MRI accurately measures cortical thickness. Courtesy of Prof. Brad Dickerson.

in 'preventative' clinical trials of various types of interventions," states Dickerson. His team also aims to compare this MRI signature with other biomarkers of $\mathrm{AD}$, to assess its strengths and weaknesses, and to determine whether it will be of use in studies of strategies to delay the onset of symptomatic AD.

Katy Malpass

Original article Dickerson, B. C. et al. Alzheimer-signature MRI biomarker predicts AD dementia in cognitively normal adults. Neurology 76, 1395-1402 (2011) 